\title{
Probing non-Abelian statistics of Majorana fermions in ultracold atomic superfluid
}

\author{
Shi-Liang Zhu, ${ }^{1}$ L.-B. Shao, ${ }^{2}$ Z. D. Wang, ${ }^{2}$ and L.-M. Duan ${ }^{3}$ \\ ${ }^{1}$ Laboratory of Quantum Information Technology and SPTE, \\ South China Normal University, Guangzhou, China \\ ${ }^{2}$ Department of Physics and Center of Theoretical and Computational Physics, \\ The University of Hong Kong, Pokfulam Road, Hong Kong, China \\ ${ }^{3}$ Department of Physics and MCTP, Michigan University, Ann Arbor, USA
}

\begin{abstract}
We propose an experiment to directly probe the non-Abelian statistics of Majorana fermions by braiding them in an s-wave superfluid of ultracold atoms. We show different orders of braiding operations give orthogonal output states that can be distinguished through Raman spectroscopy. Realization of Majorana states in an s-wave superfluid requires strong spin-orbital coupling and a controllable Zeeman field in the perpendicular direction. We present a simple laser configuration to generate the artificial spin-orbital coupling and the required Zeeman field in the dark state subspace.
\end{abstract}

PACS numbers: 03.75.Mn, 03.75.Lm, 05.30.Pr, 03.67.Lx

Quantum statistics is a fundamental concept in physics that distinguishes fermions from bosons. The discovery of the fractional quantum Hall (FQH) effects opens up the possibility to study exotic quasi-particles beyond bosons and fermions, which are called anyons [1, 2]. There are two types of anyons, with Abelian or nonAbelian statistics. For Abelian anyons, the winding of one quasi-particle around another gives a phase factor to the wave function which is a fraction of $2 \pi$. For NonAbelian anyons, a winding of one quasi-particle around another is associated with a unitary transformation in the subspace of degenerate ground states [3]. Such unitary transformations form a representation of the braid group where the elements in general are non-commutative. Recently, the systems with non-Abelian anyons have attracted considerable interest as candidates for realization of fault-tolerant quantum computation [4, 5$]$.

Probing quantum statistics is a challenge for both Abelian and non-Abelian anyons. Recent theoretical works suggest that interference experiments in a well designed point contact geometry may serve as a probe of Abelian [6] or non-Abelian anyons [7] in the FQH system. Besides of the quasi-particles in the FQH system, a vortex excitation in $p_{x}+i p_{y}$ superconductor traps a zero-energy bound state which represents a kind of self-conjugate particles called Majorana fermions [8]. Majorana fermions have the feature of non-Abelian statistics and have raised significant interest in condensed matter physics [5, 8 12 ]. Several inspiring proposals have been suggested very recently to realize Majorana fermions in different materials [10, 11]. A scheme to observe Majorana fermions has also been proposed in the p-wave superfluid state of ultracold atoms [12].

In this paper, we propose an experiment to probe nonAbelian statistics of the Majorana fermions with an ultracold atomic gas in the s-wave superfluid state. In contract to the p-wave superfluid state, which still remains to be realized experimentally due to challenge associated with instability of the p-wave Feshbach resonance [13], the s-wave superfluid state is stable and has been observed in many experiments 14]. It has been noted recently that an s-wave superfluid with spin-orbital (SO) coupling emulates properties of the p-wave superfluid [10, 11, 15, 16]. Realization of Majorana fermions in an s-wave superfluid state requires SO coupling and a controllable Zeeman field on the effective spins [1]. For ultracold atoms, an effective SO coupling can be generated through gradient of spatially varying Berry phase induced from laser beams [17-19]. We propose a scheme to realize both SO coupling and the required perpendicular Zeeman field on the effective spin, which a laser configuration compatible with the current experiment and significantly simpler than the previous proposal [19]. The Majorana fermions can be adiabatically moved around each other through control of the pinning potentials from focused laser beams. We construct explicitly two composite braiding operations $A$ and $B$, for which the different orders of braiding operations $A B$ and $B A$, acting on the initial vacuum, give orthogonal output states that can be distinguished through the Raman spectroscopy. The proposed experiment thus provides a promising approach to directly probe the fundamental non-Abelian statistics of the anyons. Realization of Majorana fermions has been proposed in other systems [10, 11], however, for typical condensed matter materials, it is very challenging to directly demonstrate the non-Abelian statistics of the Majorana fermions by braiding them in space. The proposed realization of Majorana fermions in an s-wave atomic superfluid therefore offers a unique opportunity to adiabatically rotate them in space with focused laser beams to observe the non-Abelian fractional statistics, which is the most interesting property associated with these exotic quasi-particles.

We consider a cloud of ultracold fermionic atoms with three relevant spin components in the ground-state manifold, label as $|1\rangle,|2\rangle,|3\rangle$ (e.g., they can be taken as the lowest three Zeeman levels of ${ }^{6} \mathrm{Li}$ atoms near the broad s-wave Feshbach resonance). To generate the 
effective SO coupling, we apply three traveling wave laser beams in the $x$ and $y$ directions which couple the atomic levels $|1\rangle,|2\rangle,|3\rangle$ resonantly to the excited state $|e\rangle$ through the standard tripod configuration as shown in Fig. 1 [17-19]. The single-particle Hamiltonian for each atom takes the form $H_{s}=\mathbf{p}^{2} / 2 m+H_{e j}$, where $\mathbf{p}$ denotes the momentum operator and $m$ is the atomic mass. The light-atom interaction Hamiltonian $H_{e j}$ is given by $H_{e j}=\hbar \sum_{j=1}^{j=3}\left(\Omega_{j}|e\rangle\langle j|+\right.$ h.c. $)=\hbar \Omega|e\rangle\langle B|+$ h.c., where the corresponding Rabi frequencies take the form $\Omega_{1}=\Omega \sin \beta \cos \phi \mathrm{e}^{-i k x}, \Omega_{2}=\Omega \cos \beta \mathrm{e}^{-i k y}$, and $\Omega_{3}=\Omega \sin \beta \sin \phi \mathrm{e}^{i k x}$ with $k$ the wave number of the light, $\Omega=\sqrt{\left|\Omega_{1}\right|^{2}+\left|\Omega_{2}\right|^{2}+\left|\Omega_{3}\right|^{2}}$, and the bright state $|B\rangle \equiv\left(\Omega_{1}^{*}|1\rangle+\Omega_{2}^{*}|2\rangle+\Omega_{3}^{*}|3\rangle\right) / \Omega$. We assume uniform plane wave laser beams so that $\Omega, \beta, \phi$ are all constants and the values of $\beta, \phi$ will be specified below. The diagonalization of $H_{e j}$ yields two orthogonal dark states $\left|D_{1}\right\rangle=\sin \phi \mathrm{e}^{-i k(y-x)}|1\rangle-\cos \phi \mathrm{e}^{-i k(y+x)}|3\rangle,\left|D_{2}\right\rangle=$ $\cos \beta \cos \phi \mathrm{e}^{-i k(y-x)}|1\rangle-\sin \beta|2\rangle+\cos \beta \sin \phi \mathrm{e}^{-i k(y+x)}|3\rangle$, which are not coupled to the excited state $|e\rangle$ and thus immune to spontaneous emission. We assume the atoms are initially pumped to these dark states and they remain in the dark states as long as the typical velocity $v$ of the atoms satisfy the condition $\eta v k \ll \Omega$ [20], where the prefactor $\eta \approx 1.3$ for the isotropic SO coupling discussed below. In typical experiments, the wave number $k \approx 10^{7} \mathrm{~m}^{-1}$ and the Rabi frequency $\Omega$ of the order of magnitude $2 \pi \times 20 \mathrm{MHz}$ can be readily obtained, so the condition $\eta v k \ll \Omega$ is fully satisfied for ultracold atoms with the typical atomic velocity about several centimeters per second. The probability of non-adiabatic excitation, estimated by $(\eta v k / \Omega)^{2}$, is negligible (about $10^{-6}$ ). In a resonant coupling configuration as shown in Fig. 1a, the effective atomic spontaneous emission rate is reduced from the original rate $\gamma_{s}$ by a factor of the nonadiabatic excitation probability and thus estimated by $\gamma_{s}(\eta v k / \Omega)^{2}$, where is at a few $\mathrm{Hz}$ level. So the atomic spontaneous emissions induced by these laser beams are negligible if the experimental time is within $100 \mathrm{~ms}$. The effective atomic spontaneous emission rate can be further reduced if one uses off-resonant coupling configuration or increases the Rabi frequency $\Omega$ with more laser power. Under the above condition, we can project the Hamiltonian $H_{s}$ to the subspace spanned by these two dark states, and the projected Hamiltonian takes the form

$$
H_{s o}=\frac{\left(p_{x}-A_{x}\right)^{2}}{2 m}+\frac{\left(p_{y}-A_{y}\right)^{2}}{2 m},
$$

where $A_{x}=-\hbar k \cos \beta \sigma_{x}, A_{y}=(\hbar k / 2) \sin ^{2} \beta \sigma_{y}+$ $(\hbar k / 2)\left(1+\cos ^{2} \beta\right)$. In the derivation, we have assumed $\phi=\pi / 4$ and defined an effective spin with the basis vector $|\uparrow\rangle=\left(\left|D_{1}\right\rangle+i\left|D_{2}\right\rangle\right) / \sqrt{2}$ and $|\downarrow\rangle=\left(i\left|D_{1}\right\rangle+\right.$ $\left.\left|D_{2}\right\rangle\right) / \sqrt{2}$. The Pauli matrices $\sigma_{x}, \sigma_{y}$ are defined in association with these spin basis vectors. Equation (11) describes the atom in a spin-dependent synesthetic gauge field [17-19], and a spin-independent version of this gauge field has been demonstrated in recent experiments [21]. To get the standard form of the SO coupling, we choose the value of $\beta$ to satisfy $\sin ^{2} \beta=2 \cos \beta=2(\sqrt{2}-1)$ and observe the system in the moving frame with the velocity $v_{y}=(\hbar k / 2 m)\left(1+\cos ^{2} \beta\right)$, so that we can drop the constant term $(\hbar k / 2)\left(1+\cos ^{2} \beta\right)$ in $A_{y}$.

The effective Hamiltonian $H_{s o}$ has the required SO coupling, but we still need to generate a perpendicular Zeeman field on the effective spin $|\uparrow\rangle,|\downarrow\rangle$ with the coupling term $h_{z} \sigma_{z}$. For this purpose, we apply two additional laser beams that couple the states $|1\rangle$ and $|3\rangle$ to the excited state $|e\rangle$ off-resonantly with a large detuning $\Delta_{d}$ (see Fig.1(a)), with the corresponding Rabi frequencies denoted by $\Omega_{1 z}=i\left|\Omega_{z}\right| \mathrm{e}^{-i k x}$ and $\Omega_{3 z}=\left|\Omega_{z}\right| \mathrm{e}^{i k x}$. With the driving $\left|\Omega_{1 z}\right|,\left|\Omega_{3 z}\right| \ll \Delta_{d}$, it leads to a perturbation Hamiltonian given by $H_{z}=-i \hbar \Omega_{p} e^{2 i k x}|1\rangle\langle 3|+$ h.c, where $\Omega_{p}=\left|\Omega_{z}\right|^{2} / \Delta_{d}$ [22]. We assume $\Omega_{p} \ll \Omega$, so the Hamiltonian $H_{p}$ can not pump the atoms outside of the dark state space spanned by the $|\uparrow\rangle$ and $|\downarrow\rangle$ levels, however, it splits the degeneracy of these two levels and leads to a coupling Hamiltonian of the form $H_{z}=\left(\hbar \Omega_{p} \cos \beta\right) \sigma_{z}$ in the dark state space, which gives exactly the effective Zeeman field term $h_{z} \sigma_{z}$ with $h_{z}=\hbar \Omega_{p} \cos \beta$. The Zeeman field $h_{z} / \hbar$ can be continuously tuned from zero to about $2 \pi \times 50 \mathrm{kHz}$ (enough for our later applications), and under this condition, the requirement $\Omega_{p} \ll \Omega$ is well satisfied with the off-resonant excitation probability $\left(\Omega_{p} / \Omega\right)^{2}<10^{-5}$. Note that in this scheme we need only two laser beams shined from the same direction as $\Omega_{1}, \Omega_{2}$ to create the required Zeeman field. This significantly simplifies the experimental requirement compared with the scheme in Ref. [19] which needs five additional laser beams and more complicated optical configuration to create the Zeeman field.
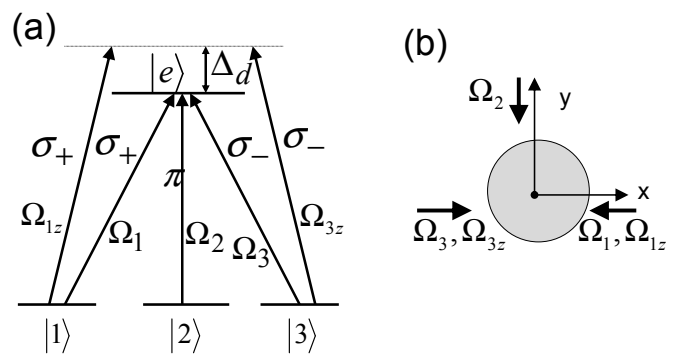

FIG. 1: Schematic representation of the light-atom interaction configuration for generation of the effective Hamiltonian with SO coupling and a perpendicular Zeeman field. (a) The relevant atomic levels and the coupling Rabi frequencies to corresponding laser beams with specified polarizations $\sigma_{+}$, $\sigma_{-}$, or $\pi$. (b) The spatial configuration and propagating direction of the laser beams.

Now we have the effective single-particle Hamiltonian $H_{s o}+H_{z}$ of the right form. The atomic interaction is described by the s-wave scattering. In the second quantization formalism, it is described by the Hamiltonian $H_{\text {int }}=\sum_{i<j} \int g_{i j} \Psi_{i}^{\dagger}(\mathbf{r}) \Psi_{j}^{\dagger}(\mathbf{r}) \Psi_{j}(\mathbf{r}) \Psi_{i}(\mathbf{r}) \mathbf{d}^{3} \mathbf{r}$, 
where $\Psi_{i}(\mathbf{r})$ denote the fermionic field operators, $i, j$ are summarized over the three spin components $1,2,3$, and $g_{i j}$ are proportional to the s-wave scattering lengths in the corresponding channel. In the s-wave superfluid state, the Hamiltonian $H_{\text {int }}$ can be replaced by the effective mean-field BCS type Hamiltonian $H_{B C S}=$ $\sum_{i<j} \int \Delta_{i j}(\mathbf{r}) \Psi_{j}(\mathbf{r}) \Psi_{i}(\mathbf{r}) \mathbf{d}^{3} \mathbf{r}+$ h.c., where $\Delta_{i j}(\mathbf{r}) \equiv$ $g_{i j}\left\langle\Psi_{i}^{\dagger}(\mathbf{r}) \Psi_{j}^{\dagger}(\mathbf{r})\right\rangle$ correspond to the superfluid order parameters. The typical s-wave pairing potential $\Delta_{i j}$ in experiments is about $2 \pi \times 3.5 \mathrm{kHz}$ 14], which is much smaller than $\Omega$, so the interaction Hamiltonian $H_{B C S}$ can not pump the atoms outside of the dark-state subspace. Under this condition, we can project $H_{B C S}$ to the dark-state subspace, and the projected Hamiltonian $H_{P}$ takes the form $H_{P}=\int \Delta(\mathbf{r}) \Psi_{\uparrow}(\mathbf{r}) \Psi_{\downarrow}(\mathbf{r}) \mathbf{d}^{3} \mathbf{r}+$ h.c. , where $\Delta$ is a linear superposition of $\Delta_{12}, \Delta_{13}, \Delta_{23}$ with the superposition coefficients determined from the transition matrix between $|1\rangle,|2\rangle,|3\rangle$ and $|\uparrow\rangle$, $|\downarrow\rangle,|B\rangle$. If we write the wave-function $\Phi(\mathbf{r})$ as $\Phi(\mathbf{r})=\left[u_{\uparrow}(\mathbf{r}), u_{\downarrow}(\mathbf{r}), v_{\downarrow}(\mathbf{r}), v_{\uparrow}(\mathbf{r})\right]^{T}$ in the basis specified by the operators $\left(\Psi_{\uparrow}(\mathbf{r}), \Psi_{\downarrow}(\mathbf{r}), \Psi_{\downarrow}^{\dagger}(\mathbf{r}),-\Psi_{\uparrow}^{\dagger}(\mathbf{r})\right)^{T}$, the Schrodinger equation for the total Hamiltonian $H=$ $H_{s o}+H_{z}+H_{P}$ with eigen-energy $E$ takes the form of the Bogoliubov-de Gennes (BdG) equation

$$
\left(\begin{array}{cc}
H_{s o}+H_{z} & \Delta(\mathbf{r}) \\
\Delta^{*}(\mathbf{r}) & -\sigma_{y}\left(H_{s o}^{*}+H_{z}^{*}\right) \sigma_{y}
\end{array}\right) \Phi(\mathbf{r})=E \Phi(\mathbf{r}) .
$$

If we introduce $\tau_{x, y, z}$ to denote Pauli matrices in the Nambu spinor space, the Hamiltonian $H$ can be written in the form

$$
\begin{aligned}
H= & {\left[\frac{p^{2}}{2 m}-\mu\right] \tau_{z}+\alpha\left(\sigma_{x} p_{x}+\sigma_{y} p_{y}\right) \tau_{z}+h_{z} \sigma_{z} } \\
& +\left(\tau_{x} \operatorname{Re} \Delta-\tau_{y} \operatorname{Im} \Delta\right),
\end{aligned}
$$

where the laser induced SO coupling rate $\alpha=-\hbar k(\sqrt{2}-$ 1). All the constant potentials have been included into the definition of the chemical potential $\mu$ which is selfconsistently determined from the particle density. For ultracold atoms in a global spin-independent confining trap $V(\mathbf{r})$, under the local density approximation, the global trap can also be included into the definition of the chemical potential with the substitution $\mu \rightarrow \mu-V(\mathbf{r})$.

The Hamiltonian $H$ in Eq.(3) has been investigated in the context of a recent proposal for realization of nonAbelian anyons in a solid state heterostructure consisting of a superconductor, a 2D electron gas with Rashba SO coupling, and a magnetic insulator [1]]. It is known that for this Hamiltonian, a Majorana fermion zero mode emerges from a vortex core if the parameters in the Hamiltonian satisfy the condition $h_{z}>h_{c}=\sqrt{\mu^{2}+|\Delta|^{2}}$ [11, 16]. For ultracold atoms, $h_{c}$ is comparable with the Fermi energy $E_{f}$ since the chemical potential $|\mu| \sim E_{f}$ and the pairing potential $|\Delta| \sim 0.35 E_{f}$. The condition $h_{z}>h_{c}$ can be easily satisfied since we assume $h_{z} / \hbar$ can be tuned up to $2 \pi \times 50 \mathrm{kHz}$, while $E_{f} / \hbar$ is around $2 \pi \times 10$ $\mathrm{kHz}$ [14]. A vortex is needed for emergency of the Majorana fermion in a central potential because the Hamiltonian has the particle-hole symmetry [11]. Vortices in atomic superfluid can be created with several methods and have been observed in many experiments [23, 24]. For instance, the required angular momentum associated with the vortices can come from initial rotation of the atomic cloud [23] or be imprinted from nonzero orbital angular momentum of laser beams [24].

We now turn to the important question of probing nonAbelian statistics associated with Majorana fermions. For an unambiguous demonstration of the non-Abelian statistics, we need to construct two braiding operations $A$ and $B$. When applied to the initial state, the composite braidings $A B$ and $B A$ give orthogonal output states which can be distinguished through the experimental detection. In the following, we explicitly construct these braiding operations for Majorana fermions and discuss the experimental detection of the output states.

Let us consider $2 N$ Majorana fermions confined in $2 N$ vortex cores, denote by $\gamma_{i}(i=1,2, \cdots, 2 N)$. The Majorana fermions can be combined pairwise to form $N$ complex fermionic states $c_{j}=\gamma_{2 j}+i \gamma_{2 j-1}$. Each fermionic state can be occupied or unoccupied and therefore the ground state is $2^{N}$-fold degenerate. If we exchange one Majorana fermion $j$ with its nearest neighbor $j+1$ by adiabatically moving the pinning potential, we get an unitary operation $\tau_{j}=\exp \left(\pi \gamma_{j+1} \gamma_{j} / 4\right)=$ $\left(1+\gamma_{j+1} \gamma_{j}\right) / \sqrt{2}$ acting on the $2^{N}$-dimensional degenerate Hilbert space [9]. The elementary interchange operations $\tau_{j}(j=1,2, \cdots, 2 N-1)$ form a complete set of generators for the braid group. To demonstrate the non-Abelian statistics, we need at least four Majorana fermions due to existence of the superselection rule. For four Majorana fermions, they combine into two complex fermions $c_{1}, c_{2}$. The ground state has degeneracy four, but the Hamiltonian $H$ conserves parity of the fermion number, and the even-number subspace spanned by $\left\{|0\rangle, c_{1}^{\dagger} c_{2}^{\dagger}|0\rangle\right\}$ is decoupled from the odd-number subspace spanned by $\left\{c_{1}^{\dagger}|0\rangle, c_{2}^{\dagger}|0\rangle\right\}$. At low temperature (temperature significantly below the superfluid gap), the initial state is typically a vacuum state $|0\rangle$ [12], and in the even-number subspace $\left\{|0\rangle, c_{1}^{\dagger} c_{2}^{\dagger}|0\rangle\right\}$, only two of the three generators $\tau_{1}, \tau_{2}, \tau_{3}$ are independent with

$$
\tau_{1}=\tau_{3}=\frac{1}{\sqrt{2}}\left(\begin{array}{cc}
1-i & 0 \\
0 & 1+i
\end{array}\right), \tau_{2}=\frac{1}{\sqrt{2}}\left(\begin{array}{cc}
1 & -i \\
-i & 1
\end{array}\right) .
$$

From these generators, we construct two composite braiding operations

$$
A=\tau_{1} \tau_{2} \tau_{1}^{-1}, B=\tau_{1}^{-1} \tau_{2} \tau_{1}^{-1}
$$

with the property

$$
A B=i \sigma_{z}, \quad B A=-i \sigma_{x},
$$


where $\tau_{1}^{-1}$ represents a braiding along the reverse direction. With the initial state $|0\rangle$, the braiding operations $A B$ and $B A$ yield two orthogonal output states, either $i|0\rangle$ or $-i c_{1}^{\dagger} c_{2}^{\dagger}|0\rangle$, demonstrating the non-Abelian nature of the anyons. During braiding, we need to fulfill the adiabaticity. The gap to the first excited state in the vortex core is estimated by $\omega_{0} \sim \Delta^{2} / E_{f} \sim 2 \pi \times 1.2 \mathrm{kHz}$. If the braiding time $t_{b}$ is of the order of tens of ms, the nonadiabatic excitation probability estimated by $1 /\left(\omega_{0} t_{b}\right)^{2}$ is negligible.

The two output states $i|0\rangle$ and $-i c_{1}^{\dagger} c_{2}^{\dagger}|0\rangle$ can be distinguished through the Raman (or r.f.) spectroscopy [25]. For the state $c_{1}^{\dagger} c_{2}^{\dagger}|0\rangle$, we have two fermionic atoms at zero energy trapped at the vortex cores. We can apply a Raman $\pi$-pulse to resonantly bring these zero-energy atoms to a different hyperfine state $|D\rangle$ [12], which is outside of Feshbach resonance and thus only weakly interacting with other atoms. If we choose the Rabi frequency of the Raman pulse to have a Gaussian shape $\Omega_{R}=\Omega_{0} \exp \left(-r^{2} / w^{2}\right) \exp \left(-\omega_{0}^{2} t^{2}\right)$ (where $-t_{f} \leq t \leq t_{f}$ and the center of the vortex core is at $r=0)$, it will not break up the Cooper-pairs or excite other quasiparticles at the vortex core which are detuned typically by an energy scale comparable with $|\Delta|$. It is estimated that the error is about $4 \times 10^{-4}$ and thus negligible under typical experimental parameters $\Delta=2 \pi \times 3.5 \mathrm{kHz}, \omega_{0}=0.35 \Delta$, $\Omega_{0}=1.7 \omega_{0}, t_{f}=5 / \omega_{0}$ and $w=1.5 \mu \mathrm{m}$. If the two laser beams for the Raman pulse propagate along different directions, it also applies a momentum kick to the target atoms to push them outside of the atomic cloud. These target atoms in a different hyperfine state $|D\rangle$ can then be detected with the standard quantum jump technique.

In summary, we have proposed an experimental scheme to create and observe Majorana fermions in an s-wave superfluid of ultracold fermionic atoms with laser induced SO coupling. The non-Abelian statistics of the Majorana fermions can be directly probed through laser-controlled braiding and detection based on the Raman spectroscopy.

This work was supported by the NSF of China (No 10974059), the SKPBR of China (Nos.2011CB922104 and 2007CB925204), the RGC of Hong Kong, the DARPA OLE Program under ARO Award W911NF0710576, and the AFOSR MURI program.
[2] F. Wilczek, Phys. Rev. Lett. 48, 1144 (1982); F. Wilczek, Fractional Statictics and Anyon Superconductivity (World Scientific, Singapore, 1990).

[3] G. Moore and N. Read, Nucl. Phys. B 360, 362 (1991).

[4] A. Kitaev, Annals Phys. 303, 2 (2003).

[5] C. Nayak et al., Rev. Mod. Phys. 80, 1083 (2008).

[6] C. L. Kane, Phys. Rev. Lett. 90, 226802 (2003); C. Chamon et al., Phys. Rev. B 55, 2331 (1997).

[7] A. Stern and B. I. Harperin, Phys. Rev. Lett. 96, 016802 (2006); S. Das Sarma, M. Freedman, and C. Nayak, ibid. 94, 166802 (2005); D. E. Feldman and A. Kitaev, ibid. 97, 186803 (2006).

[8] N. Read and D. Green, Phys. Rev. B 61, 10267 (2000).

[9] D. A. Ivanov, Phys. Rev. Lett. 86, 268 (2001).

[10] L. Fu and C. L. Kane, Phys. Rev. Lett. 100, 096407 (2008).

[11] J. D. Sau et al., Phys. Rev. Lett. 104, 040502 (2010); Phys. Rev. B 82, 214509 (2010).

[12] S. Tewari et al., Phys. Rev. Lett. 98,010506 (2007).

[13] J. P. Gaebler et al., Phys. Rev. Lett. 98, 200403 (2007).

[14] W. Ketterle and M. W. Zwierlein, arXiv:0801.2500

[15] C. Zhang et al., Phys. Rev. Lett. 101, 160401 (2008); A. Kubasiak et al., Europhys. Lett. 92, 46004 (2010).

[16] M.Sato et al., Phys. Rev. Lett. 103, 020401 (2009).

[17] G. Juzeliunas and P. Ohberg, Phys. Rev. Lett. 93, 033602 (2004); J. Ruseckas et al., ibid. 95, 010404 (2005); P. Zhang et al., Eur. Phys. J. D 36, 229 (2005).

[18] S. L. Zhu et al., Phys. Rev. Lett. 97, 240401 (2006); X.J.Liu et al., ibid., 98, 026602 (2006); T. D. Stanescu, B. Anderson, and V. Galitski, Phys. Rev. A 78, 023616 (2008).

[19] C. Zhang, Phys. Rev. A 82, 021607(R) (2010).

[20] This condition is obtained by requiring the off-diagonal coupling matrix element of the Hamiltonian $H_{s}$ between the dark states and the bright state much smaller than the energy difference $\hbar \Omega$.

[21] Y. J. Lin et al., Nature (London) 462, 628 (2009).

[22] There is also a constant a.c.-Stark shift term $\left(\left|\Omega_{z}\right|^{2} / \Delta_{d}\right)(|1\rangle\langle 1|+| 3\rangle\langle 3|)$ associated with the laser beams $\Omega_{1 z}, \Omega_{3 z}$. However, this constant frequency shift can be easily canceled with a corresponding frequency offset of the laser beam $\Omega_{2}$ applied to the level $|2\rangle$, so it has no physical effect on the ground levels.

[23] M.W.Zwierlein et al., Nature (London) 435, 1047 (2005).

[24] M.F.Andersen et al., Phys. Rev. Lett. 97, 170406 (2006).

[25] T.-L. Dao et al., Phys. Rev. Lett. 98, 240402 (2007); A. Schirotzek et al., Phys. Rev. Lett. 101, 140403 (2008).

[1] R. B. Laughlin, Phys. Rev. Lett. 50, 1395 (1983). 